\title{
Design of Low Power and High Linearity Gm-C Low Pass Filters
}

\author{
Xiushan $\mathrm{Wu}^{1, *}$, Yanzhi Wang ${ }^{1}$, Qingying $\mathrm{Chen}^{1}$, Xingzhou Jiang ${ }^{2}$, Renyuan Tong ${ }^{1}$, Jianqiang $\operatorname{Han}^{1}$ and Siguang $\mathrm{An}^{1}$ \\ ${ }^{1}$ College of Mechanical and Electrical Engineering, China Jiliang University, Hangzhou, China \\ ${ }^{2}$ Zhejiang Longyou electric power engineering, Longyou, Zhejiang, China \\ ${ }^{*}$ Corresponding author
}

\begin{abstract}
This paper presents two kinds fully differential operational transconductance amplifier (OTA) circuit, one is a flipped voltage follower circuit structure (OTA1), the other is a source follow circuit structure (OTA2). The two OTAs' transconductance can all be accurately set by the control voltage. Two Gm-C Low-Pass Filters are designed respectively based on the two OTAs, the filters are the 4th order Butterworth low-pass filter by 2 Biquads, and their cut-off frequency are $100 \mathrm{kHz}$. Simulation is designed in Cadence using SMIC 0.13 $\mu \mathrm{m}$ CMOS technology, the supply voltage is $1.2 \mathrm{~V}$, the bias current is set to $7.2 \mu \mathrm{A}$ and the common mode voltage is set to $0.6 \mathrm{~V}$. The results show that the transconductance values have good linearity in specified range of the input voltage, and the $\mathrm{Gm}-\mathrm{C}$ filters can all meet the design requirements.
\end{abstract}

\section{Keywords-component; OTA; Gm-C; low-pass filter; biquad}

\section{INTRODUCTION}

In the rapid development of the wireless communication field, the transceiver system with high performance is a hot research area in recent years [1]. The filter is one of the most important part in this system, is the key module to solve transceiver performance, with low cost and low power CMOS technology to increase the feasibility of the design of high performance filter. So it is very important to design a low power and high performance filter with low power consumption.

The main type of continuous time filters is Active-RC, MOSFET-C and Gm-C, which are widely used in the wireless $\mathrm{RF}$ transceiver. Active-RC filter [2] has better linearity and larger dynamic range than others, but it requires the operational amplifier's bandwidth is larger, which will increase the power consumption of the circuit, and it cannot precisely adjust the frequency characteristics by the capacitance switch or resistance, which limits its application. MOSFET-C filter[3] is use the MOSFET resistor that work in the triode region instead of resistance, it is an extension of active $\mathrm{RC}$, at the expense of the linearity and dynamic range cost to achieve precise adjustment of the frequency characteristics, and has a serious distortion, power consumption, that is also equal to active RC. The Gm-C filter[4] using OTA as active elements without low impedance output stage, the designed filter's bandwidth can reach the unity gain bandwidth and frequency can be continuously adjusted, the power required is minimal. At the same time, through the precisely adjust the transconductance can achieve high precision transmission characteristics. Despite the linear is poor when OTA work in open loop, it can use some linear measures to improve. The design of a low power high linear high performance low pass filter is using this Gm-C structure, which can precisely transform the frequency by regulating the OTA.

In this paper, we use two OTA structures to build fourorder Butterworth low-pass filters respectively, whose cut-off frequency is $100 \mathrm{kHz}$, and compare the effect of two kinds.

\section{The Circuit Design of OTA}

The performance of the transconductor directly determines the performance of the filter. To the transconductor, wide linearity is necessary. At present, a large number of linearization techniques to improve the linear range of transconductor have been proposed [5-11], but these linearization techniques are mostly at the expense of gain, noise and power consumption. At the same time, the transconductor's finite DC gain and parasitic high-order point make the Gm-C integrator in the unit gain bandwidth shift 90 degrees at phase, it caused serious effect about the filter transfer function's precision. Therefore, increasing the output impedance of the transconductor to reduce the frequency of the dominant pole, improving the DC gain, and reducing the number of transconductor's internal nodes to improve the transconductor parasitic higher order poles is key of a transconductor design[12]. In addition, the transconductance value can be effectively adjusted is also a guarantee of achieving high precision filter's transfer function.

In order to reduce power consumption to improve linearity, this paper will use the MOS transistors in the linear region as a transconductance to achieve voltage to current conversion. For the NMOS transistor operating in the linear region, the drain current is:

$$
I_{D}=\mu_{n} C_{o x} \frac{W}{L}\left[\left(V_{G S}-V_{T H}\right) V_{D S}-\frac{1}{2} V_{D S}^{2}\right]
$$

Therefore, the NMOS transistor transconductance in the linear region is

$$
g_{m}=\frac{\partial I_{D}}{\partial V_{G S}}=\mu_{n} C_{o x} \frac{W}{L} V_{D S}
$$


If can be seen from formula (2) that the MOS tube based on linear region has good linear degree, and the transconductance value can be directly controlled by $V_{\mathrm{DS}}$. In order to be able to precisely control the $V_{\mathrm{DS}}$, we have used two methods: the first introduces the circuit structure of flipped voltage follower, as shown in Figure I; the second introduces the circuit structure of source follower, as shown in Figure II.

\section{A. The Structure of the OTA1}

Flipped voltage follower has small quiescent current, but can transmit large current to the load, and low power advantages. It is widely used in low voltage low power analog signal processing circuit. At the same time, the Pseudo differential operational amplifier can avoid the influence of tail current and obtain wider linear input range, besides, it is suitable for low voltage applications. Based on the above proposed a high performance OTA structure shown in Figure I.

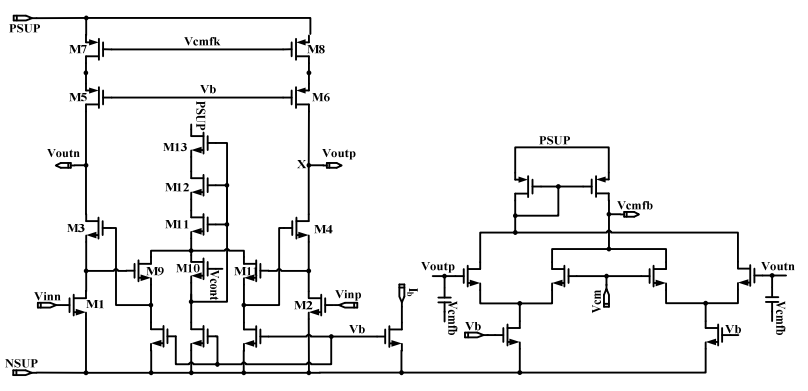

FIGURE I. STRUCTURE OF THE OTA1

The structure uses the NMOS M1 and M2 work in the linear region as the input stages, because of the linear relationship between the large signal transconductance of $\mathrm{M} 1 / \mathrm{M} 2$ and $V_{\mathrm{DS}}$, so the circuit's linearity depends mainly on the $V_{\mathrm{DS}}$ of M1/M2. The common source common gate tube M3 and M4 are used to stabilize the $V_{\mathrm{DS}}$ of the input tube which is not affected by the large input current variation, while the introduction of FVF and feedback loop (M3 and M9) further ensure the stability of $V_{\mathrm{DS}}$. The stable negative feedback loop formed by the M10, M12 and the bias current ensures that the current flowing through the NMOS transistor M10 is constant. Ignore the effect of bulk effect and short channel effect, $V_{\mathrm{SG}, \mathrm{M} 10}$ will remain unchanged. The output node $\mathrm{X}$ is a low resistance state:

$$
r_{o}=\frac{1}{g_{m 10} g_{m 12} r_{o 10}}
$$

$g_{\mathrm{m} 10} / g_{\mathrm{m} 12}$ is small signal transconductance of NMOS transistor M10/M12, $r_{\mathrm{o} 10}$ is small signal output impedance of M10, the node impedance $r_{\mathrm{o}}$ in about $100 \Omega$ or less. Because of the output node in the low impedance state, making the circuit can produce a larger current, but irrigation into the MOS transistor current is limited by the bias current $I_{\mathrm{b}}$, exhibits amplifier's characteristics, and achieves low power. So when the M9, M10 and M11 complete matching and bias current is the same, it can make the leakage dc voltages of the input M1and M2 are equal to the conditioning voltage $V_{\text {tune, which }}$ can accurately change circuit transconductance values by changing the $V_{\text {tune }}$.

M3 and M9, M4 and M11 constitute a negative feedback loop, respectively, to enhance the stability of the input tube $V_{\mathrm{DS}, \mathrm{M} 1}$ and $V_{\mathrm{DS}, \mathrm{M} 2}$. At the same time, the feedback loop can improve the output impedance of OTA1, and the leakage resistance of transistor M3 can approximate as:

$$
r_{\text {out }}=g_{m 3} g_{m 9} r_{o 1} r_{o 3} r_{o 9}
$$

It can be seen that M9 also plays a magnifying effect in the feedback loop, so that the output impedance increases by $g_{\mathrm{m} 9} r_{\mathrm{o}} 9$ times. The M9 needs to work in the saturation area to ensure the change range of the input voltage and the adjustable voltage. M13 and M14 are used to reduce the gate source voltage to ensure that the circuit requirements of the other transistor working area. M5 M8 as an active load, ensure the high output impedance. At the same time, in order to ensure smaller output current loss and larger adjustable range, lower voltage is needed to work at the input tube in the linear region.

\section{B. The Structure of the OTA2}

The circuit structure of the transconductor is using the fully differential mode circuit with two input and two output ends, and the common mode feedback to suppress common mode gain. The specific circuit is shown in Figure II. In order to increase input range and offset the second-order effect, the MN9 and MN10 in sub-threshold region, MN1 and MN2 in the linear region, MN3-MN6 is source follower to provide a suitable bias voltage for the MN9 and MN10. There are two operational amplifier, their role is to make the drain voltage of the MN1/MN2 equal to $V_{\text {tune. }}$. From the formula (2), we can see that changing the value of $V_{\text {tune }}$ can directly change the size of the transconductance, and then change the cutoff frequency of the $\mathrm{Gm}-\mathrm{C}$ filter can achieve the purpose of precise control.

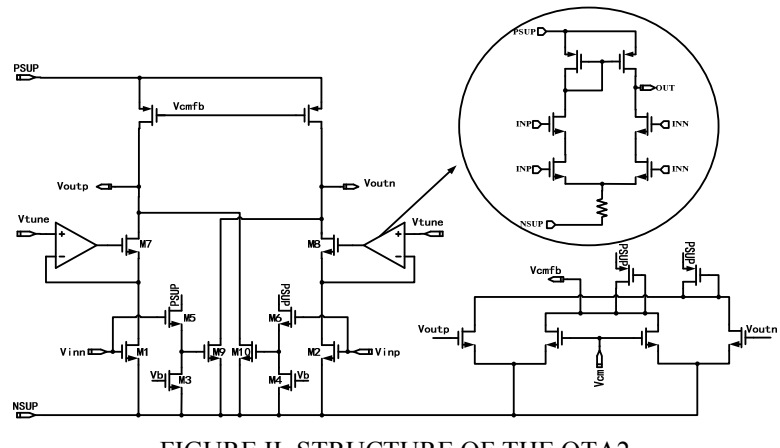

FIGURE II. STRUCTURE OF THE OTA2

\section{THE DESIGN OF THE FILTER}

Due to the effect of the cascade circuit at all levels is very small, it can easily adjust the device parameters and the loads at all levels of filter which formed by cascade circuit are basically the same. In addition, his adaptive circuit can make adjustment accuracy of the filter center frequency is very high, suitable for the design of a higher order filter. Therefore, two Gm-C LowPass Filters are designed respectively based on the two OTAs 
to realize the 4th-order Butterworth low-pass filter by 2 Biquads. Check the data can be known, the design of a $100 \mathrm{kHz}$ 4th-order Butterworth low pass filter, the cascade parameters' value of each section as shown in Figure III, and the structure of OTA filter is shown in Figure IV.

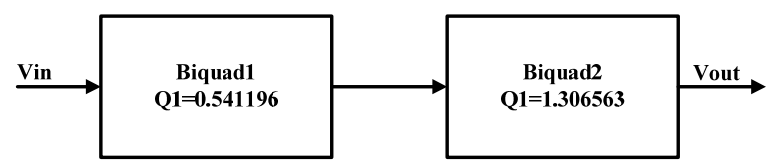

FIGURE III. CASCADE PARAMETERS' VALUE OF EACH SECTION

The transfer function for each level is

$$
H(s)=\frac{V_{\text {out }}(s)}{V_{\text {in }}(s)}=\frac{\frac{g_{m 2} g_{m 1}}{C_{2} C_{1}}}{S_{2}+S\left(\frac{g_{m 3}}{C_{2}}\right)+\left(\frac{g_{m 2} g_{m 1}}{C_{2} C_{1}}\right)}
$$

For $\mathrm{g}_{\mathrm{m} 1}=\mathrm{g}_{\mathrm{m} 2}=\mathrm{g}_{\mathrm{m} 3}=\mathrm{g}_{\mathrm{m} 4}=\mathrm{g}_{\mathrm{m}}$, then

$$
\begin{aligned}
& w_{0}=\sqrt{\frac{g_{m}^{2}}{C_{1} C_{2}}} \\
& Q=\sqrt{\frac{C_{2}}{C_{1}}}
\end{aligned}
$$

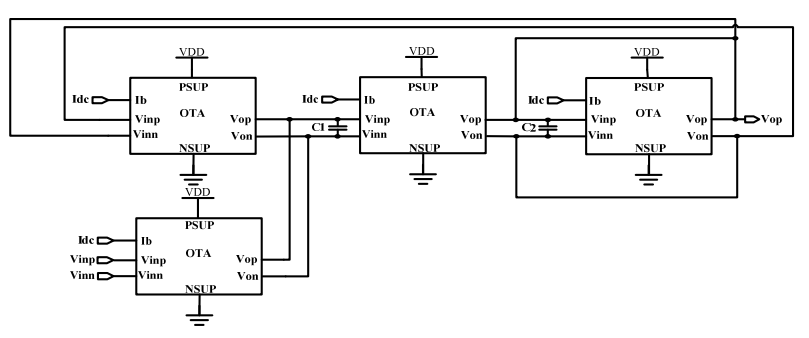

FIGURE IV. STRUCTURE OF THE FILTER

\section{RESUlTS AND DisCUSSION}

The low pass filter circuit is designed by SMIC $0.13 \mu \mathrm{m}$ CMOS process in Cadence. The supply voltage of the system is $1.2 \mathrm{~V}$, the common mode voltage is $0.6 \mathrm{~V}$, and the bias current is $7.2 \mu \mathrm{A}$.

The constant degree of two kinds OTA's transconductance value in a certain range of input voltage reflects the transconductor linearity. The simulation results of two kinds transconductance values as shown in Figure V. It is obvious to show that the transconductance value has a adjustable range from $10 \mu \mathrm{S}$ to $60 \mu \mathrm{S}$ along with the control voltage $V_{\text {tune }}$ change from $50 \mathrm{mV}$ to $300 \mathrm{mV}$. Among them, when the $V_{\text {tune }}$ changes from $50 \mathrm{mV}$ to $100 \mathrm{mV}$, the transconductance value of OTA1 (Gm1) changes less than $1 \%$; when the $V_{\text {tune }}$ changes from 150
$\mathrm{mV}$ to $300 \mathrm{mV}, \mathrm{Gm} 1$ changes less than $3 \%$; when the $V_{\text {tune }}$ changes from $50 \mathrm{mV}$ to $100 \mathrm{mV}$, the transconductance value of OTA2 (Gm2) changes less than $3 \%$; when the $V_{\text {tune }}$ changes from $150 \mathrm{mV}$ to $300 \mathrm{mV}, \mathrm{Gm} 2$ changes less than $0.7 \%$.From the above, through the control of $V_{\text {tune }}$ to select the corresponding transconductance, and the low-pass filter can achieve high accuracy.

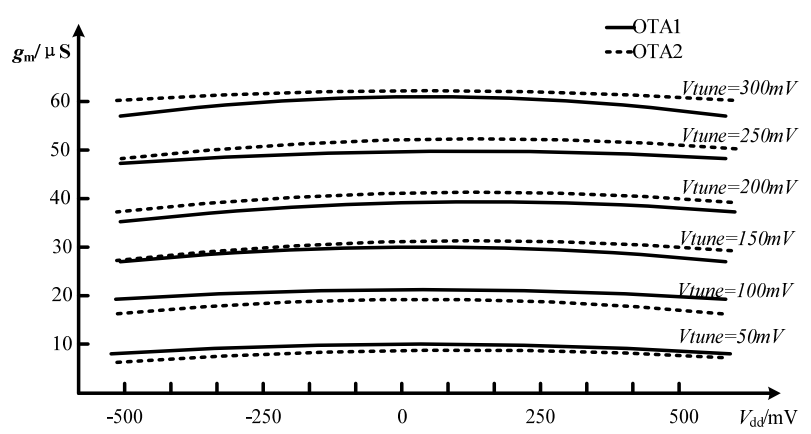

FIGURE V. SIMULATION OF TWO KINDS TRANSCONDUCTANCE VALUES

In order to reduce the capacitance value, we use $V_{\text {tune }}=50 \mathrm{mV}$, so $\mathrm{g}_{\mathrm{m} 1}=\mathrm{g}_{\mathrm{m} 2}=\mathrm{g}_{\mathrm{m} 3}=\mathrm{g}_{\mathrm{m} 4}=\mathrm{g}_{\mathrm{m}}=9.8 \mu \mathrm{S}$. Figure VI is the simulation results of the four order Butterworth low pass filters' frequency response, and their cut-off frequency error is within $\pm 1 \%$.

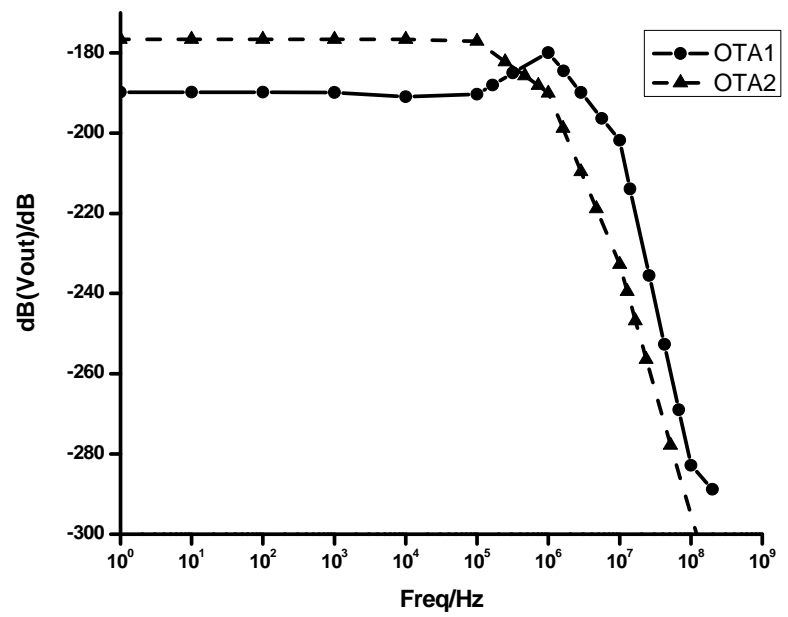

FIGURE VI. SIMULATION OF THE FILTER FREQUENCY RESPONSE

\section{CONCLUSIONS}

In this paper, two kinds of topology optimization method, has realized the low power consumption and high linear transconductor, and design the cut-off frequency is $100 \mathrm{kHz}$ fourth-order Butterworth low-pass filter with $0.13 \mu \mathrm{m}$ CMOS technology. According to the simulation results show that the two structures of transconductor are able to achieve great performance, and both of them have own advantages in the control the voltage change. At the same time, based on the two kinds, respectively, we have constructed 4th Butterworth low pass filter successfully, and achieved the desired effect. 


\section{ACKNOWLEDGMENT}

This research was financially supported by the National Natural Science Foundation of Zhejiang (LY14F040004), Zhejiang Key Discipline of Instrument Science and technology (No.JL130102 and JB150518) and the National Natural Science Foundation of China (51407172 and 61076110).

\section{REFERENCES}

[1] Lucía Acosta, Mariano Jiménez, Ramón G. Carvajal et al.Highly Linear Tunable CMOS Gm-C Low-Pass Filter[J].IEEE Transactions on circuits and systems,2009:2145-2158.

[2] Siva V.Thyagarajan,Shanthi Pavan,and Prabu Sankar.Active-RC Filters Using the Gm-Assisted OTA-RC Technique[J].IEEE Journal of solidstate circuits,2011: 1522-1533.

[3] Chen Fangxiong,Lin Min,Ma Heping et al.An asymmetric MOSFET-C band-pass filter with on-chip charge pump auto-tuning[J].Journal of Semiconductors,2009,30(8):1-5.

[4] Lewinski A,Silva M J.A $30 \mathrm{MHz}$ fifth order elliptic low-pass CMOS filter with $65 \mathrm{~d}$ B spurious-free dynamic range[J].IEEE Trans on Circuits Syst I,2007,54(3):469-480.

[5] Ajayan.K.R,Bhat.N,Linear transconductor with flipped voltage follower in $130 \mathrm{~nm}$ CMOS[J].Analog Integrated Circuits Signal Process.2010,63(2):321-327.

[6] Martinez-Heredia.J.M,Torralba.A,Enhanced source-degenerated CMOS differential transconductor[J], Microelectronics Journal,2011,42(2):396402 .

[7] Szczepanski.S,Pankiewicz.B,Koziel.S,Programmable feedforward linearized CMOS OTA for fully differential continuous-time filter design[J].Circuit Theory and Applications.2010,38(9):885-899.

[8] Farouk.T,Mohieldin.AN,Khalil.AH, A low-voltage low-power CMOS fully differential transconductor with mobility reduction compensation[J],Microelectronocs Journal.2012,43(1):69-76.

[9] Pedro.M,Galán.J,Sánchez-Rodríguez.T,et al.A linear compact tunable transconductor for Gm-C applications, Analog Integrated Circuits and Signal Process.2012,72(2):351-361.

[10] Lo.T.Y,Hung.C.C,.Lo.C.H, Linear low voltage nano-scale CMOS transconductor, Analog Integrated Circuits and Signal Process.2011,66(1):1-7.

[11] Kuo.KC,Chen SY,Tseng.SM. High linear transconductor for multiband CMOS receiver[C], IEEE Asia pacific conference on circuits and systems, 2012:535-538.

[12] Ferreira LHC, Sonkusale SR. G(m) enhancement for bulk-driven subthreshold differential pair in nanometer CMOS process. IEEE Subthreshold Microelectronics Conference. 2012: 1-3.G. Eason, B. Noble, and I. N. Sneddon, "On certain integrals of Lipschitz-Hankel type involving products of Bessel functions," Phil. Trans. Roy. Soc. London, vol. A247, pp. 529-551, April 1955. 